\title{
Edge Detection Method based on Lifting B-Spline Dyadic Wavelet
}

\author{
Zhibin Hu, Caixia Deng*, Yunhong Shao, and Cui Wang \\ Department of Mathematics, Harbin University of Science and Technology, Harbin, 150080, China
}

\begin{abstract}
Aiming at the problem of discontinuous edge details in the wavelet transform modulus maxima edge detection algorithm, the shortcomings of the algorithm are addressed by improving the smoothness of the wavelet function and selecting appropriate wavelet filters. The order of vanishing moments of a wavelet function determines the ability of the wavelet to approximate smooth function. Therefore, this paper focuses on improving the order of vanishing moments of the B-spline dyadic wavelet, giving a new lifting scheme and lifting parameters, and realizing a B-spline dyadic wavelet filter with high-order vanishing moments, symmetry, and compact support. At the same time, an edge detection algorithm based on lifting the B-spline dyadic wavelet is proposed. The experimental results show that the algorithm can effectively suppress noise and display the continuous details of the image edges.
\end{abstract}

Keywords: dyadic lifting scheme; high order vanishing moments; histogram equalization; edge detection

(Submitted on December 15, 2018; Revised on January 12, 2019; Accepted on February 17, 2019)

(C) 2019 Totem Publisher, Inc. All rights reserved.

\section{Introduction}

The edge of a picture is generally defined as a region in which the gray value of the image changes dramatically [1]. In the image, the mutation point of the gray value is located on the boundary of the important target, and edge detection finds the mutation points. The edges of the image are widely used in the fields of image segmentation, recognition, and detection of part cracks [2].

Traditional image edge detection methods include Canny operator, Sobel operator, and Laplacian operator. These algorithms are easy to implement and have a wide range of applications, but they are sensitive to noise and have poor noise reduction effect [3]. In the past 30 years, wavelet analysis has rapidly developed into a new analytical processing tool [4]. Wavelet analysis has good localization features in both time and frequency domain, so it has good effect image processing. For example, the filter can extract the texture information of the picture and also reduce the impact of noise on the image [5]. The wavelet transform modulus maxima algorithm is one of the most commonly used edge detection methods. This algorithm can not only reduce the impact of noise, but also clearly distinguish the contour features of the image. However, the edge details of images are easily lost, resulting in discontinuity of edges and reduction of details [6].

By analyzing the shortcomings of the modulus maxima algorithm of wavelet transform, we can see that the wavelet filter is one of the factors affecting the results of the algorithm. The selection of wavelet filters often has the following characteristics: orthogonality, vanishing moments, symmetry, and tight support. However, it is very difficult to find orthogonal wavelets with the above properties at the same time. Therefore, it is necessary to extend the orthogonal conditions and study it on the basis of non-orthogonality. The common non-orthogonal wavelets are biorthogonal wavelets, dyadic wavelets, etc. Due to the down-sampling and non-translation invariance of biorthogonal wavelet transform, the accuracy of image processing is not good [7]. The dyadic wavelet transform has translation invariance without down sampling, and it achieves good performance in image processing accuracy [8-9]. In 2002, Abdukirim proposed a dyadic lifting scheme, which can not only improve the order of vanishing moments of dyadic wavelets filter, but also obtain the dyadic wavelet filter with many good properties [10]. The order of wavelet vanishing moments determines the ability of the wavelet to approximate smooth functions. In the wavelet modulus maxima edge detection algorithm, the smoothness of the

\footnotetext{
* Corresponding author.

E-mail address: dengcx2001@hotmail.com
} 
wavelet function affects the continuity of its edge. Therefore, a new lifting scheme of dyadic wavelet is proposed by analyzing the dyadic lifting scheme and B-spline dyadic wavelet. Abdukirim's lifting method is a special case of this new lifting scheme. A new dyadic wavelet filter with high order vanishing moment, symmetry, and compact support is realized by using this method. Secondly, in the process of edge detection of the dyadic wavelet transform modulus maxima algorithm, some detail information will be lost in the process of denoising because of the noise in the real edge of the image. In order to promote the contrast of the picture, the histogram equalization method is used to enhance the image so as to retain more edge details [11]. Therefore, the edge detection results obtained by this algorithm show more image details and continuous edges than the traditional methods.

\section{Dyadic Lifting Scheme}

Some of the concepts discussed in this section will be used in the later chapters, which are detailed in the literature [4, 8].

\subsection{The Dyadic Wavelets Filter}

Let function $\hat{\psi}(\omega)$ be the Fourier transform of the function $\psi(t) \in L^{2}(R)$, which is defined as

$$
\hat{\psi}(\omega)=\int_{-\infty}^{+\infty} \psi(t) e^{-i \omega t} \mathrm{~d} t
$$

For $A, B>0$, there is $A \leq \sum_{j=-\infty}^{+\infty}\left|\hat{\psi}\left(2^{j} \omega\right)\right|^{2} \leq B$, (a.e. $\omega \in R$ ), and then $\psi(t)$ is called a dyadic wavelet function.

The dyadic wavelet transform of $f(t)$ can be expressed in the following convolution form:

$$
W f\left(2^{-j}, u\right)=\int_{-\infty}^{+\infty} f(t) \sqrt{2^{j}} \overline{\psi\left(-2^{j}(u-t)\right)} \mathrm{d} t=\sqrt{2^{j}}\left(f \times \overline{\psi\left(-2^{j} t\right)}\right)(u), j \in Z, u \in R
$$

Where

$$
\psi_{2^{-j}, u}(t)=\sqrt{2^{j}} \psi\left(2^{j}(t-u)\right)
$$

Definition 2.1 Let the filters $\hat{h}(\omega), \hat{g}(\omega), \hat{\tilde{h}}(\omega)$, and $\hat{\tilde{g}}(\omega)$ satisfy the following equation:

$$
\begin{aligned}
& \hat{\varphi}(2 \omega)=\hat{h}(\omega) \hat{\varphi}(\omega), \quad \hat{\tilde{\varphi}}(2 \omega)=\hat{\tilde{h}}(\omega) \hat{\tilde{\varphi}}(\omega) \\
& \hat{\psi}(2 \omega)=\hat{g}(\omega) \hat{\varphi}(\omega), \quad \hat{\tilde{\psi}}(2 \omega)=\hat{\tilde{g}}(\omega) \hat{\tilde{\varphi}}(\omega)
\end{aligned}
$$

Such that

$$
\hat{\tilde{h}}(\omega) \hat{h}^{*}(\omega)+\hat{\tilde{g}}(\omega) \hat{g}^{*}(\omega)=2
$$

Where

$$
\begin{aligned}
& \hat{h}(\omega)=\sum_{k} h[k] e^{-i \omega k} / \sqrt{2}, \quad \hat{g}(\omega)=\sum_{k} g[k] e^{-i \omega k} / \sqrt{2} \\
& \hat{\tilde{h}}(\omega)=\sum_{k} \tilde{h}[k] e^{-i k \omega} / \sqrt{2}, \quad \hat{\tilde{g}}(\omega)=\sum_{k} \tilde{g}[k] e^{-i k \omega} / \sqrt{2}
\end{aligned}
$$

Then, the filters $\hat{h}(\omega), \hat{g}(\omega), \hat{\tilde{h}}(\omega)$, and $\hat{\tilde{g}}(\omega)$ are dyadic wavelet filters. Equation (3) is called the Dyadic Perfect Reconstruction (DPR) condition, and the symbols ' ' ' and '*' denote the meaning of duality and conjugate, respectively. 


\subsection{B-Spline Dyadic Wavelet}

In this section, we introduce the B-spline dyadic wavelet filter constructed by Mallat [12]. The $m$ times B-spline function $\varphi(t)$ is a convolution of the characteristic function $\chi_{[0,1]}$ with itself $m+1$ times. The Fourier transform of function $\varphi(t)$ is denoted by

$$
\hat{\varphi}(\omega)=\left(\frac{\sin (\omega / 2)}{\omega / 2}\right)^{m+1} e^{\frac{-i \varepsilon \omega}{2}}, \varepsilon=\left\{\begin{array}{ll}
1, & m=2 n \\
0, & m=2 n+1
\end{array}, n \in Z\right.
$$

From Equation (1), we get

$$
\hat{h}(\omega)=\sqrt{2} \frac{\hat{\varphi}(2 \omega)}{\hat{\varphi}(\omega)}=\sqrt{2}\left(\cos \frac{\omega}{2}\right)^{m+1} e^{\frac{-i \varepsilon \omega}{2}}
$$

In order to construct the spline dual scaling function $\hat{\tilde{\varphi}}(\omega)$ and wavelet function $\hat{\tilde{\psi}}(\omega)$, we take $\hat{\tilde{h}}(\omega)=\hat{h}(\omega)$. A high pass filter $\hat{g}(\omega)$ can be obtained from the low pass filter $\hat{h}(\omega)$. It follows that

$$
\hat{g}(\omega)=(-i)^{\tau} \sqrt{2} e^{\frac{-i(2-\tau) \omega}{2}}\left(\sin \frac{\omega}{2}\right)^{r}, \quad \tau= \begin{cases}1, & r=1 \\ 0, & r=2\end{cases}
$$

From the dyadic perfect reconstruction condition (DPR), we can get

$$
\hat{\tilde{g}}(\omega)=(-i)^{\tau} \sqrt{2} e^{\frac{-i(2-\tau) \omega}{2}}\left(\sin \frac{\omega}{2}\right)^{2-r} \sum_{l=0}^{m}\left(\cos \frac{\omega}{2}\right)^{2 l}
$$

By the definition of the dyadic wavelet function $\hat{\psi}(\omega)$, we have

$$
\hat{\psi}(\omega)=\left(-\frac{\omega}{4}\right)^{r} e^{-i\left(\frac{(2+\varepsilon-\tau) \omega}{4}-\frac{\pi}{2} \tau\right)}\left(\frac{\sin (\omega / 4)}{\omega / 4}\right)^{m+r+1}
$$

Therefore, it follows that Equations (7) to (9) are dyadic wavelet filters.

\subsection{Dyadic Lifting Scheme and Vanishing Moments}

Definition 2.2 Let the wavelet $\psi(t) \in L^{2}(R)$, if $\int_{-\infty}^{+\infty} t^{k} \psi(t) \mathrm{d} t=0$ for $0 \leq k<p$, and then $\psi(t)$ has $p$ vanishing moments.

Lemma 2.1 Let the Fourier transform $\hat{\psi}(\omega)$ of the wavelet $\psi(t)$ have $p$ multiple zeros at $\omega=0$, and then the following conclusions are mutually equivalent:

$$
\left.\frac{\mathrm{d}^{k} \hat{\psi}(\omega)}{\mathrm{d} \omega^{k}}\right|_{\omega=0}=0,0 \leq k<p,\left.\quad \frac{\mathrm{d}^{k} \hat{g}(\omega)}{\mathrm{d} \omega^{k}}\right|_{\omega=0}=0,0 \leq k<p, \quad \int_{-\infty}^{+\infty} t^{k} \psi(t) \mathrm{d} t=0,0 \leq k<p
$$

In order to construct the B-spline dyadic wavelet filters easily and improve vanishing moment orders, we obtain the following lifting theorem, which can obtain the general expression of the dyadic wavelet filters.

Theorem 2.1 If the filters $\hat{h}^{0}(\omega), \hat{g}^{0}(\omega), \hat{\tilde{h}}^{0}(\omega)$, and $\hat{\tilde{g}}^{0}(\omega)$ are the initial dyadic wavelet filters, we get the expression of the lifted filters $\hat{h}^{l}(\omega), \hat{g}^{l}(\omega), \hat{\tilde{h}}^{l}(\omega)$, and $\hat{\tilde{g}}^{l}(\omega)$ as follows: 


$$
\left\{\begin{array}{l}
\hat{h}^{l}(\omega)=\hat{\lambda}(\omega) \hat{h}^{0}(\omega) \\
\hat{g}^{l}(\omega)=\hat{\lambda}^{-1}(\omega) \hat{g}^{0}(\omega)-\hat{s}(\omega) \hat{h}^{0}(\omega) \\
\hat{\tilde{h}}^{l}(\omega)=\hat{\lambda}^{-1^{*}}(\omega) \hat{\tilde{h}}^{0}(\omega)+\hat{s}^{*}(\omega) \hat{\tilde{g}}^{0}(\omega) \\
\hat{\tilde{g}}^{l}(\omega)=\hat{\lambda}^{*}(\omega) \hat{\tilde{g}}^{0}(\omega)
\end{array}\right.
$$

Then, the lifted dyadic wavelet filters $\hat{h}^{l}(\omega), \hat{g}^{l}(\omega), \hat{\tilde{h}}^{l}(\omega)$, and $\hat{\tilde{g}}^{l}(\omega)$ are also dyadic wavelet filters, where $\hat{\lambda}(\omega) \neq 0,(\forall \omega \in R)$, and $\hat{s}(\omega)$ are free parameters, and Equation (11) is called the B-spline dyadic wavelet lifting scheme. The symbol ' $l$ ' is denoted as the meaning of lifting.

Proof The filters $\hat{h}^{0}(\omega), \hat{g}^{0}(\omega), \hat{\tilde{h}}^{0}(\omega)$, and $\hat{\tilde{g}}^{0}(\omega)$ are dyadic wavelet filters, which satisfy the condition of DPR. Using Equations (3) and (11), we can prove the conclusion.

The following corollary will explain how to increase the order of vanishing moments by changing the parameters in the dyadic lifting scheme.

\section{Corollary 2.1}

- If the initial filter $\hat{g}^{0}(\omega)$ satisfies $\hat{g}^{0}(0) \neq 0$ in Theorem 2.1, then a sufficient condition for $\hat{g}^{l}(0)=0$ is $\hat{s}(0)=\hat{\lambda}(0) \hat{g}^{0}(0) / \hat{h}^{0}(0)$.

- If the initial dyadic wavelet high pass filter $\hat{g}^{0}(\omega)$ has $p$ vanishing moments, the lifted dyadic wavelet high pass filter $\hat{g}^{l}(\omega)$ has at least $p+1$ vanishing moments if and only if $\hat{s}(\omega)$ satisfies

$$
\left.\frac{\mathrm{d}^{k} \hat{s}(\omega)}{\mathrm{d} \omega^{k}}\right|_{\omega=0}=0,0 \leq k<p,\left.\quad \frac{\mathrm{d}^{p} \hat{s}(\omega)}{\mathrm{d} \omega^{p}}\right|_{\omega=0}=\left.\left(\frac{\hat{\lambda}(\omega)}{\hat{h}^{0}(\omega)} \frac{\mathrm{d}^{p} \hat{g}^{0}(\omega)}{\mathrm{d} \omega^{p}}\right)\right|_{\omega=0} \neq 0
$$

Proof The Corollary (1) is easy to get, and we need only to prove Corollary (2). Since the initial wavelet $\psi^{0}$ has $p$ vanishing moments, it follows that

$$
\left.\frac{\mathrm{d}^{k} \hat{g}^{0}(\omega)}{\mathrm{d} \omega^{k}}\right|_{\omega=0}=0,0 \leq k<p,\left.\frac{\mathrm{d}^{p} \hat{g}^{0}(\omega)}{\mathrm{d} \omega^{P}}\right|_{\omega=0} \neq 0
$$

According to Theorem 2.1, it follows that $\hat{g}^{l}(\omega)=\hat{\lambda}(\omega) \hat{g}^{0}(\omega)-\hat{s}(\omega) \hat{h}^{0}(\omega)$. Take the derivative of both sides of the above equation, and then

$$
\frac{\mathrm{d}^{k} \hat{g}^{l}(\omega)}{\mathrm{d} \omega^{k}}=\sum_{m=0}^{k}\left(\begin{array}{l}
k \\
m
\end{array}\right) \frac{\mathrm{d}^{k-m} \hat{\lambda}(\omega)}{\mathrm{d} \omega^{k-m}} \frac{\mathrm{d}^{m} \hat{g}^{0}(\omega)}{\mathrm{d} \omega^{m}}-\sum_{m=0}^{k}\left(\begin{array}{l}
k \\
m
\end{array}\right) \frac{\mathrm{d}^{m} \hat{s}(\omega)}{\mathrm{d} \omega^{m}} \frac{\mathrm{d}^{k-m} \hat{h}^{0}(\omega)}{\mathrm{d} \omega^{k-m}}
$$

If $0 \leq k<p$, use Equations (13) and (14) to get $\left.\frac{\mathrm{d}^{k} \hat{g}^{l}(\omega)}{\mathrm{d} \omega^{k}}\right|_{\omega=0}=0$.

It is clear that if $k=p$, then we obtain $\left.\frac{\mathrm{d}^{p} \hat{g}^{l}(\omega)}{\mathrm{d} \omega^{p}}\right|_{\omega=0}=0$. Therefore, the lifted dyadic wavelet filters have at least $p+1$ vanishing moments.

The lifting method of Abdukirim can be obtained by changing the parameters, so the dyadic lifting scheme is further expanded in this paper. The new lifting scheme can be used to construct dyadic wavelet filters with some properties. 
Similarly, we can get the dual lifting theorem, which is not detailed.

\section{Implementation of the Dyadic Wavelet Filter with High Order Vanishing Moments}

In this section, we construct the dyadic wavelet filters with high-order vanishing moments. First, the initial filter of the dyadic wavelet can be obtained from the content in Subsection 2.2. The following is an example of a B-spline function in case of $r=2$ and $m=3$. Combining Equations (7) through (9), we calculate the coefficients of the dyadic wavelet filters as shown in Table 1.

Table 1. The coefficients of the dyadic wavelet filter

\begin{tabular}{ccccc}
\hline$k$ & $h[k] / \sqrt{2}$ & $g[k] / \sqrt{2}$ & $\tilde{h}[k] / \sqrt{2}$ & $\tilde{g}[k] / \sqrt{2}$ \\
\hline-2 & 0.0625 & & 0.0625 & 0.015625 \\
-1 & 0.25 & & 0.25 & 0.15625 \\
0 & 0.375 & -0.25 & 0.375 & 0.734375 \\
1 & 0.25 & 0.5 & 0.25 & 2.1875 \\
2 & 0.0625 & -0.25 & 0.0625 & 0.734375 \\
3 & & & & 0.15625 \\
4 & & & & 0.015625 \\
\hline
\end{tabular}

The resulting calculation can be obtained from Table 1 , where $\hat{g}^{0}(\omega)$ satisfies

$$
\left.\frac{\mathrm{d}^{k} \hat{g}^{0}(\omega)}{\mathrm{d} \omega^{k}}\right|_{\omega=0}=0,0 \leq k<2,\left.\frac{\mathrm{d}^{2} \hat{g}^{0}(\omega)}{\mathrm{d} \omega^{2}}\right|_{\omega=0} \neq 0
$$

The initial filter $\hat{g}^{0}(\omega)$ has two vanishing moments. We can use Theorem 2.1 to construct a high pass filter $\hat{g}^{l}(\omega)$ with three or higher vanishing moments. From Corollary 2.1 , if $\hat{\lambda}(\omega)=1$, then $\hat{s}(\omega)$ must satisfy the following conditions:

$$
\left.\frac{\mathrm{d}^{k} \hat{s}(\omega)}{\mathrm{d} \omega^{k}}\right|_{\omega=0}=0,0 \leq k<2,\left.\frac{\mathrm{d}^{2} \hat{s}(\omega)}{\mathrm{d} \omega^{2}}\right|_{\omega=0}=\left.\frac{1}{\sqrt{2}} \frac{\mathrm{d}^{2} \hat{g}^{0}(\omega)}{\mathrm{d} \omega^{2}}\right|_{\omega=0}=\frac{1}{2}
$$

Considering the property of $\hat{s}(\omega)$, we can select $\hat{s}(\omega)=e^{-i \omega} \sin ^{2} \omega / 4$. Using the expression of Equation (11), we obtain the results of the coefficients of the dyadic wavelet filters after the first lifting, as shown in Table 2.

\begin{tabular}{|c|c|c|c|c|}
\hline$k$ & $h^{l}[k] / \sqrt{2}$ & $g^{l}[k] / \sqrt{2}$ & $\tilde{h}^{l}[k] / \sqrt{2}$ & $\tilde{g}^{l}[k] / \sqrt{2}$ \\
\hline-5 & & & -0.0009765625 & \\
\hline-4 & & & -0.009765625 & \\
\hline-3 & & 0.00390625 & -0.0439453125 & 0.015625 \\
\hline-2 & 0.0625 & 0.015625 & -0.0546875 & 0.15625 \\
\hline-1 & 0.25 & 0.015625 & 0.294921875 & 0.734375 \\
\hline 0 & 0.375 & -0.265625 & 0.62890625 & 2.1875 \\
\hline 1 & 0.25 & 0.4609375 & 0.294921875 & 0.734375 \\
\hline 2 & 0.0625 & -0.265625 & -0.0546875 & 0.15625 \\
\hline 3 & & 0.015625 & -0.0439453125 & 0.015625 \\
\hline 4 & & 0.015625 & -0.009765625 & \\
\hline 5 & & 0.00390625 & -0.0009765625 & \\
\hline
\end{tabular}

Table 2. The coefficients of the dyadic wavelet filter after the first lifting

From Table 2, we can see that $\hat{g}^{l}(\omega)$ satisfies

$$
\left.\frac{\mathrm{d}^{k} \hat{g}^{l}(\omega)}{\mathrm{d} \omega^{k}}\right|_{\omega=0}=0,0 \leq k<4,\left.\frac{\mathrm{d}^{4} \hat{g}^{l}(\omega)}{\mathrm{d} \omega^{4}}\right|_{\omega=0} \neq 0
$$

It is shown that the filter $\hat{g}^{l}(\omega)$ has four vanishing moments. Using Theorem 2.1 again, we can implement a filter $\hat{g}^{l l}(\omega)$ with five or more vanishing moments. From Corollary 2.1 , for $\hat{\lambda}(\omega)=1, \hat{s}(\omega)$ must satisfy 


$$
\left.\frac{\mathrm{d}^{k} \hat{s}(\omega)}{\mathrm{d} \omega^{k}}\right|_{\omega=0}=0,0 \leq k<4,\left.\frac{\mathrm{d}^{4} \hat{s}(\omega)}{\mathrm{d} \omega^{4}}\right|_{\omega=0}=\left.\frac{1}{\sqrt{2}} \frac{\mathrm{d}^{4} \hat{g}^{l}(\omega)}{\mathrm{d} \omega^{4}}\right|_{\omega=0}=\frac{9}{2}
$$

Considering the property of $\hat{s}(\omega)$, we choose $\hat{s}(\omega)=3 e^{-i \omega} \sin ^{4} \omega / 16$. Using the expression of Equation (11) again, we obtain the results of the coefficients of the dyadic wavelet filters after the second lifting, as shown in Table 3.

Table 3. The coefficients of the dyadic wavelet filter after the second lifting

\begin{tabular}{|c|c|c|c|c|}
\hline$k$ & $h^{l l}[k] / \sqrt{2}$ & $g^{l l}[k] / \sqrt{2}$ & $\tilde{h}^{l l}[k] / \sqrt{2}$ & $\tilde{g}^{l l}[k] / \sqrt{2}$ \\
\hline-7 & & & 0.00018310546875 & \\
\hline-6 & & & 0.0018310546875 & \\
\hline-5 & & -0.000732421875 & 0.00689696265625 & \\
\hline-4 & & -0.0029296875 & 0.008544921875 & \\
\hline-3 & & 0.00244140625 & -0.06866455078125 & 0.015625 \\
\hline-2 & 0.0625 & 0.0244140625 & -0.1444091796875 & 0.15625 \\
\hline-1 & 0.25 & 0.028076171875 & 0.31158447265625 & 0.734375 \\
\hline 0 & 0.375 & -0.271474375 & 0.76806640625 & 2.1875 \\
\hline 1 & 0.25 & 0.4404296875 & 0.31158447265625 & 0.734375 \\
\hline 2 & 0.0625 & -0.271484375 & -0.1444091796875 & 0.15625 \\
\hline 3 & & 0.028076171875 & -0.06866455078125 & 0.015625 \\
\hline 4 & & 0.0244140625 & 0.008544921875 & \\
\hline 5 & & 0.00244140625 & 0.00689697265625 & \\
\hline 6 & & -0.0029296875 & 0.0018310546875 & \\
\hline 7 & & -0.000732421875 & 0.00018310546875 & \\
\hline
\end{tabular}

From Table 3, we can see that $\hat{g}^{\prime \prime}(\omega)$ satisfies

$$
\left.\frac{\mathrm{d}^{k} \hat{g}^{l l}(\omega)}{\mathrm{d} \omega^{k}}\right|_{\omega=0}=0,0 \leq k<6,\left.\frac{\mathrm{d}^{6} \hat{g}^{l l}(\omega)}{\mathrm{d} \omega^{6}}\right|_{\omega=0} \neq 0
$$

It is shown that the filter $\hat{g}^{l l}(\omega)$ has six vanishing moments. Using Theorem 2.1 again, we can implement a high pass filter $\hat{g}^{l l l}(\omega)$ with seven or more vanishing moments. From Corollary 2.1 , for $\hat{\lambda}(\omega)=1, \hat{s}(\omega)$ must satisfy

$$
\left.\frac{\mathrm{d}^{k} \hat{s}(\omega)}{\mathrm{d} \omega^{k}}\right|_{\omega=0}=0,0 \leq k<6 \text { and }\left.\frac{\mathrm{d}^{6} \hat{s}(\omega)}{\mathrm{d} \omega^{6}}\right|_{\omega=0}=\left.\frac{1}{\sqrt{2}} \frac{\mathrm{d}^{6} \hat{g}^{l l}(\omega)}{\mathrm{d} \omega^{6}}\right|_{\omega=0}=\frac{405}{4}
$$

Considering the property of $\hat{s}(\omega)$, we choose $\hat{s}(\omega)=9 e^{-i \omega} \sin ^{6} \omega / 64$. In the same way, we use the expression of

\begin{tabular}{|c|c|c|c|c|}
\hline$k$ & $h^{l l l}[k] / \sqrt{2}$ & $g^{l l l}[k] / \sqrt{2}$ & $\tilde{h}^{l l l}[k] / \sqrt{2}$ & $\tilde{g}^{I l l}[k] / \sqrt{2}$ \\
\hline-9 & & & -0.000135265119634 & \\
\hline-8 & & & -0.001352651196345 & \\
\hline-7 & & 0.000137329101563 & -0.005365516412168 & \\
\hline-6 & & 0.000549316406250 & -0.009017674642299 & \\
\hline-5 & & -0.000732421875000 & 0.036551641216785 & \\
\hline-4 & & -0.005676269531250 & 0.100396777684261 & \\
\hline-3 & & -0.000305175781250 & -0.122279668149573 & 0.015625 \\
\hline-2 & 0.0625 & 0.029357910156250 & -0.391126608151978 & 0.15625 \\
\hline-1 & 0.25 & 0.036865234375000 & 0.337471444030299 & 0.734375 \\
\hline 0 & 0.375 & -0.274230957031250 & 1.094685583744140 & 2.1875 \\
\hline 1 & 0.25 & 0.428070068359375 & 0.337471444030299 & 0.734375 \\
\hline 2 & 0.0625 & -0.274230957031250 & -0.391126608151978 & 0.15625 \\
\hline 3 & & 0.036865234375000 & -0.122279668149573 & 0.015625 \\
\hline 4 & & 0.029357910156250 & 0.100396777684261 & \\
\hline 5 & & -0.000305175781250 & 0.036551641216785 & \\
\hline 6 & & -0.005676269531250 & -0.009017674642299 & \\
\hline 7 & & -0.000732421875000 & -0.005365516412168 & \\
\hline 8 & & 0.000549316406250 & -0.001352651196345 & \\
\hline 9 & & 0.000137329101563 & -0.000135265119634 & \\
\hline
\end{tabular}
Equation (11) again and obtain the coefficients of the dyadic wavelet filters after the third lifting, as shown in Table 4.

Table 4. The coefficients of the dyadic wavelet filter after the third lifting 
From Table 4, we can see that $\hat{g}^{l l l}(\omega)$ satisfies

$$
\left.\frac{\mathrm{d}^{k} \hat{g}^{l l l}(\omega)}{\mathrm{d} \omega^{k}}\right|_{\omega=0}=0,0 \leq k<8 \text { and }\left.\frac{\mathrm{d}^{8} \hat{g}^{l l l}(\omega)}{\mathrm{d} \omega^{8}}\right|_{\omega=0} \neq 0
$$

Therefore, $\hat{g}^{l l l}(\omega)$ has eight vanishing moments, and we can continue to apply Theorem 2.1 to get a dyadic wavelet filter with even higher vanishing moments.

\section{Improving the Edge Detection Algorithm}

Although the traditional edge detection methods have a wide range of applications, there are discontinuous edges and few edge details in the detected image [13-14]. Because the B-spline dyadic wavelet has high vanishing moments, it can overcome the disadvantage of discontinuous edge details. The following is an edge detection algorithm based on B-spline dyadic wavelet transform.

In 1992, Mallat proposed the edge detection method of dyadic wavelet transform [15]. Let the two variables function $\theta(x, y)$ satisfy the following two conditions:

$$
\int_{R} \int_{R} \theta(x, y) \mathrm{d} x \mathrm{~d} y=1, \lim _{x^{2}+y^{2} \rightarrow \infty} \theta(x, y)=0
$$

Then, $\theta(x, y)$ is called the smoothing function. This paper selects the cubic B-spline wavelet as a smoothing function. For the image $f(x, y)$, the convolution of the discrete smooth function $\theta_{2^{j}}(x, y)$ and the image $f(x, y)$ is used to smooth the image as follows:

$$
\left(f * \theta_{2^{j}}\right)(x, y)=\theta_{2^{j}}(x, y) * f(x, y)
$$

Where $j \in Z$ and $\theta_{2^{j}}(x, y)=\varphi_{2^{j}}(x, y)=\varphi\left(x / 2^{j}, y / 2^{j}\right) / 2^{j}$. For $\theta(x, y)$, two wavelet functions are defined as

$$
\psi^{1}(x, y)=\partial \theta(x, y) / \partial x, \psi^{2}(x, y)=\partial \theta(x, y) / \partial y
$$

At each scale $2^{j}$, let $\psi_{2^{j}}^{i}(x, y)=\psi^{i}\left(x / 2^{j}, y / 2^{j}\right) / 2^{j}$, where $i=1,2$. The dyadic wavelet transform of function $f(x, y)$ along the $x$ axis and $y$ axis directions is given as

$$
W_{2^{j}}^{1} f(x, y)=f * \psi_{2^{j}}^{1}(x, y), W_{2^{j}}^{2} f(x, y)=f * \psi_{2^{j}}^{2}(x, y)
$$

At the same scale $2^{j}$, the wavelet transform amplitude and modulus of function $f(x, y)$ are denoted as

$$
A_{2^{j}} f(x, y)=\arctan \left(\frac{W_{2^{j}}^{2} f(x, y)}{W_{2^{j}}^{1} f(x, y)}\right), M_{2^{j}} f(x, y)=\sqrt{\left|W_{2^{j}}^{1} f(x, y)\right|^{2}+\left|W_{2^{j}}^{2} f(x, y)\right|^{2}}
$$

It can be seen that the edge points of the smoothing image $f(x, y)$ correspond to the local maximum points of the modulus along the gradient vector. In other words, we can detect the local maximum points of the modulus only along the direction of amplitude, and then we can get the edge points of the image, which are the edges of the image.

In order to overcome the problem of discontinuity and reduced detail in edge detection, the edge detection algorithm of wavelet modulus maxima is improved. The edge detection algorithm is programmed using the MATLAB platform. The detail steps and flow diagram Figure 1 are as follows:

Step 1 Select the cubic dyadic wavelet function as the smoothing function. The noise image is used for dyadic wavelet 
decomposition, and then the low frequency sub image of original image is obtained.

Step 2 The low frequency image obtained from step 1 adopts the histogram equalization method to enhance the contrast of the image.

Step 3 At scale $2^{j}$, calculate the horizontal component $W_{2^{j}}^{1} f(x, y)$ and vertical component $W_{2^{j}}^{2} f(x, y)$ of the sub image.

Step 4 Calculate the modulus $M_{2^{j}} f(x, y)$ and amplitude $A_{2^{j}} f(x, y)$ of each pixel in the sub image, and then get the local maximum points of the modulus along the amplitude direction.

Step 5 The set of the local maximum points constitutes a complete edge image.

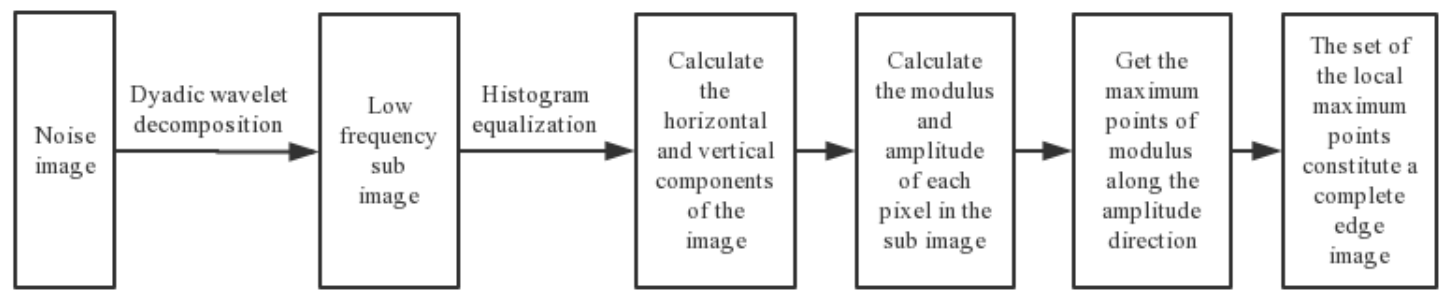

Figure 1. Basic steps of the dyadic wavelet transform edge algorithm

\section{Experimental Results and Analysis}

In order to verify the effectiveness of this method for edge detection in this paper, we add salt and pepper noise with density of 0.01 into the images of Lena, Cameraman, and Bottle. Then, we use the algorithms in references [3, 13-14] and the improved algorithm in this paper to detect the edge of the noise images. The results of edge detection are shown in Figures 2 to 4 .

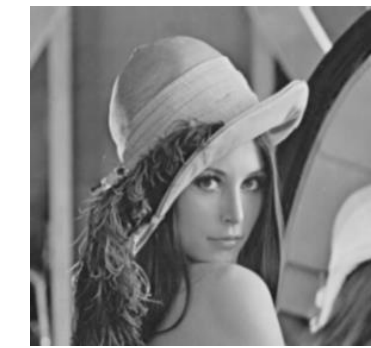

(a) Original image

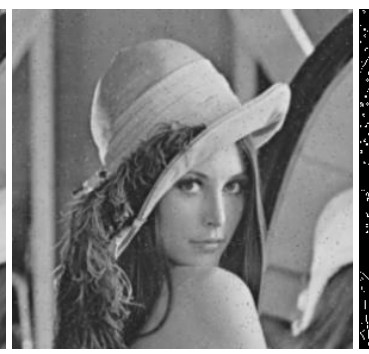

(b) Noise image

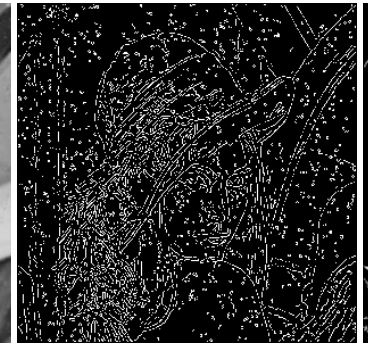

(c) The method of the Ref. [3] (d) The method of the Ref. [13]

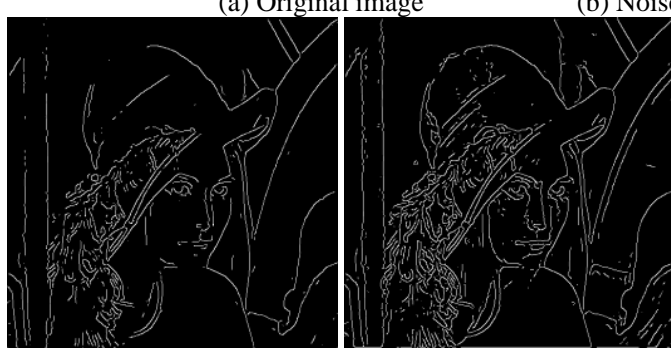

(e) The method of the Ref. [14] (f) This paper [Tab. 1]

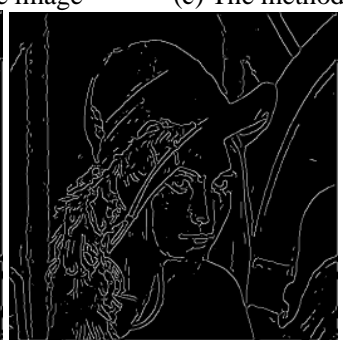

(g) This paper [Tab. 2

Figure 2. Edge detection results of Lena image

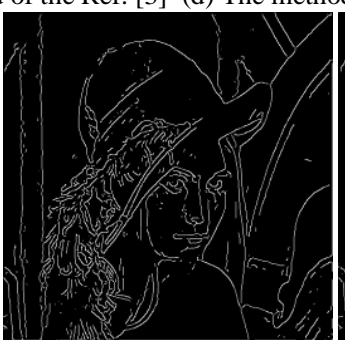

(h) This paper [Tab. 3]

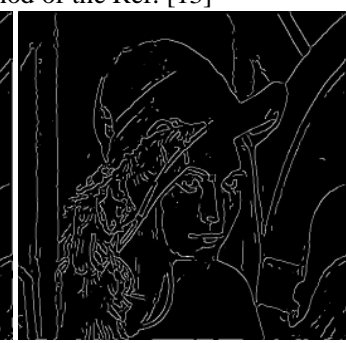

(g) This paper [Tab. 4]

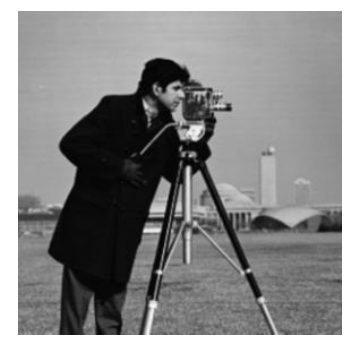

(a) Original image

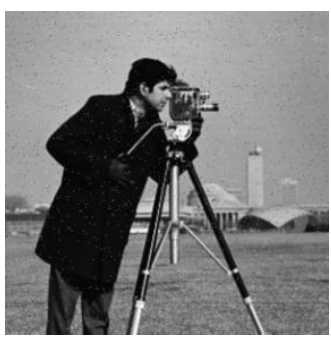

(b) Noise image

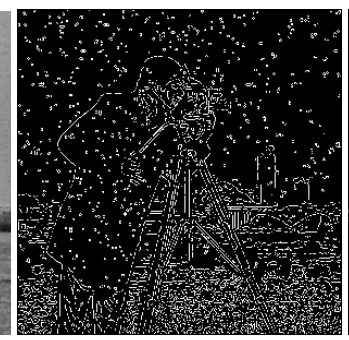

(c) The method of the Ref. [3] (d) The method of the Ref. [13] 


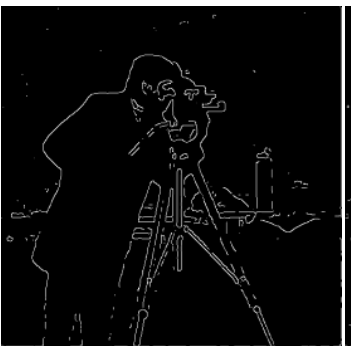

(e) The method of the Ref. [14]

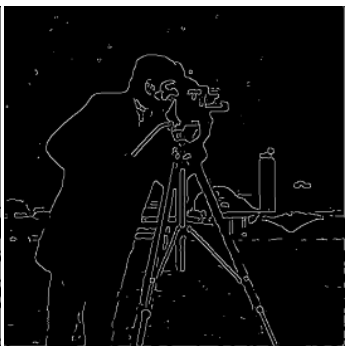

(f) This paper [Tab.1]

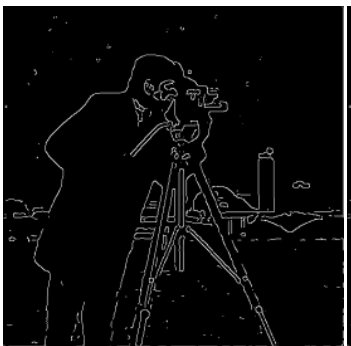

(g) This paper [Tab. 2]

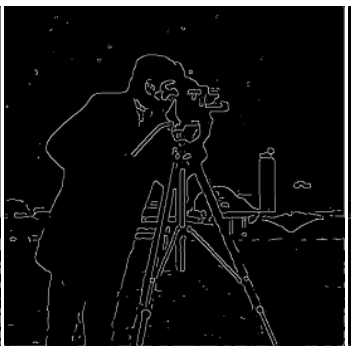

(h) This paper [Tab. 3]

Figure 3. Edge detection results of Cameraman image

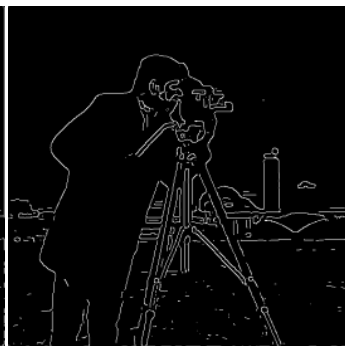

(g) This paper [Tab. 4]

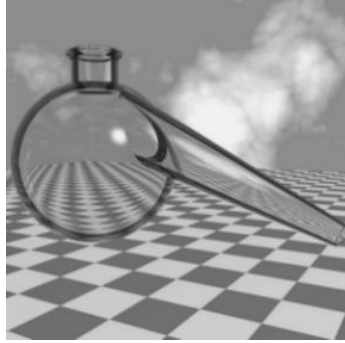

(a) Original image

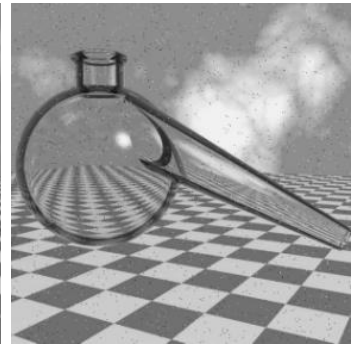

(b) Noise image

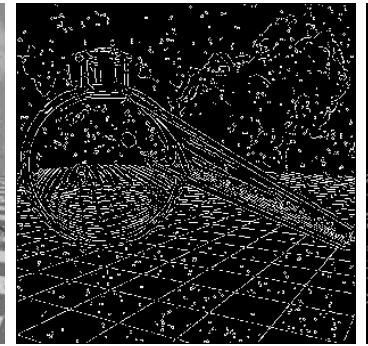

(c) The method of the Ref. [3]

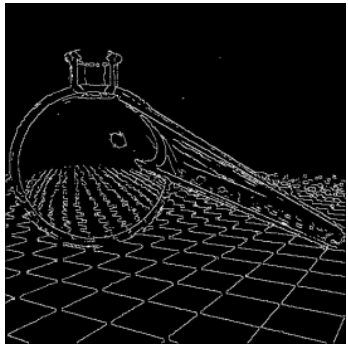

(d) The method of the Ref. [13]

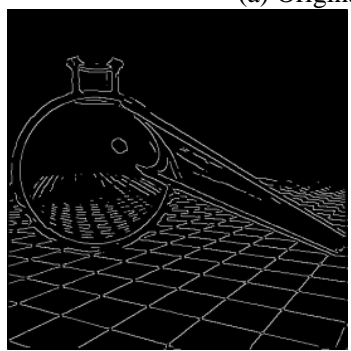

(e) The method of Ref. [14]

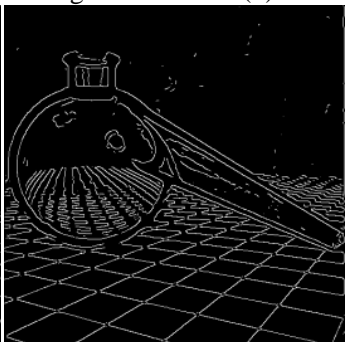

(f) This paper [Tab. 1]

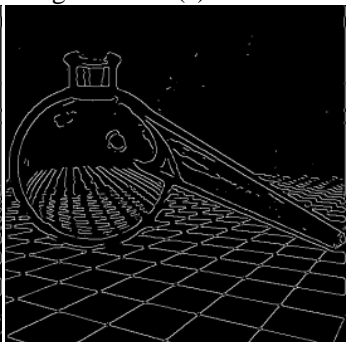

(g) This paper [Tab. 2]

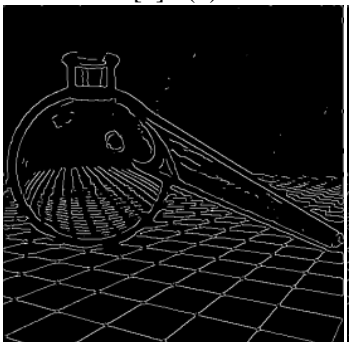

(h) This paper [Tab. 3]

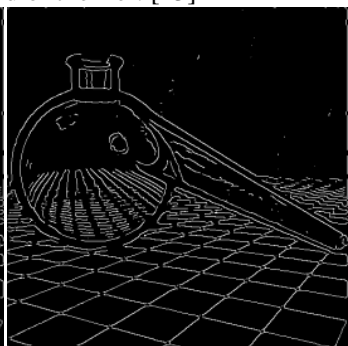

(g) This paper [Tab. 4]

Figure 4. Edge detection results of Bottle image

In order to detect the algorithm more objectively and accurately, we use the Peak Signal to Noise Ratio (PSNR) and Mean Square Error (MSE) of every image edge detection as evaluation indicators, and the results are shown in Table 5.

Table 5. The objective evaluation of edge detection algorithm

\begin{tabular}{|c|c|c|c|c|c|c|c|}
\hline \multirow{2}{*}{\multicolumn{2}{|c|}{ Algorithm }} & \multicolumn{3}{|c|}{ PSNR } & \multicolumn{3}{|c|}{ MSE } \\
\hline & & Lena & Cameraman & Bottle & Lena & Cameraman & Bottle \\
\hline The method & he Ref. [3] & 5.5359 & 5.5165 & 4.1078 & 18175.8549 & 18456.9535 & 25252.2629 \\
\hline The method & e Ref. [13] & 5.5635 & 5.5226 & 4.1485 & 18109.6056 & 17846.3587 & 25016.6498 \\
\hline \multirow{2}{*}{ This paper } & Tab. 1 & 5.5624 & 5.6395 & 4.1787 & 18065.2039 & 17747.3766 & 24843.2089 \\
\hline & Tab. 2 & 5.5705 & 5.6402 & 4.1807 & 18031.4962 & 17744.3767 & 24832.0077 \\
\hline
\end{tabular}

From Figures 2 to 4, the edge image obtained by reference [3] cannot detect the real edge under the interference of noise. Although the Canny operator is an edge detection operator based on the optimization idea, it is not necessarily optimal in practice. The method of reference [13] is easy to produce false edges, and the edge details are less. However, the detection results are better than those of reference [3]. Compared with the above methods, the algorithm of reference [14] is very rich in the details of local features has clear contour, but the edges are not continuous. Figures 2-4 (f)-(g) show the edge detection images obtained by the algorithm of this paper, which is implemented using the dyadic wavelet filter coefficients of Tables 1-4. With the improvement of the vanishing moment order, the algorithm can effectively suppress the noise of the image and display more continuous edge details of the image. In the Lena figure, for example, the lines around the hat are continuous. The house and tower in the Cameraman figure are clear and continuous. The bottle mouth and the ground mesh are continuous in the Bottle figure. Table 5 shows that the data in this paper are superior to those in references $[3,13-14]$ in the two indicators of MSE and PSNR. Therefore, the algorithm achieves good detection results and objective evaluation. It is an effective edge detection algorithm. 


\section{Conclusions}

Firstly, a new lifting scheme of dyadic is proposed in the paper. A dyadic wavelet filter is obtained by designing different lifting parameters to satisfy the actual demand. Secondly, a new lifting dyadic wavelet filter with linear phase, symmetry, compact support, and eight vanishing moments is constructed by lifting the dyadic wavelet scheme after lifting three times. Therefore, the smoothness of the dyadic wavelet is further improved, which can overcome the disadvantage of image edge discontinuity in edge detection. Finally, an improved edge detection algorithm based on dyadic wavelet transform is proposed. Compared with the traditional edge detection method, the algorithm effectively removes the noise and detects more details of the image. Meanwhile, the edge of the image has good continuity and accuracy. In summary, this paper will further expand the dyadic wavelet lifting scheme and provide a theoretical basis for image processing.

\section{Acknowledgements}

This work is supported by the National Natural Science Foundation of China (No. 51875142, 11871181).

\section{References}

1. H. Ren, S. Zhao, and J. Gruska, "Edge Detection based on Single Pixel Imaging," Optics Express, Vol. 26, No. 5, pp. 55015511,2018

2. J. C. Russ, "The Image Processing Handbook," CRC Press, 2016

3. S. Xiang and H. Zhang, "Efficient Edge-Guided Full Waveform Inversion by Canny Edge Detection and Bilateral Filtering Algorithms," Geophysical Journal International, Vol. 207, No. 2, pp. 1049-1061, 2016

4. J. Castillo, A. Mocquet, and G. Saracco, "Wavelet Transform: A Tool for the Interpretation of Upper Mantle Converted Phases at High Frequency," Geophysical Research Letters, Vol. 28, No. 22, pp. 4327-4330, 2018

5. W. C. Lin and J. W. Wang, "Edge Detection in Medical Images with Quasi High-Pass Filter based on Local Statistics," Biomedical Signal Processing and Control, Vol. 39, pp. 294-302, 2018

6. F. Y. Wang, M. Chen, and Q. S. Fei, "The Improved Method for Image Edge Detection based on Wavelet Transform with Modulus Maxima," Advances in Applied Sciences and Manufacturing, Vol. 850, No. 4, pp. 897-900, 2014

7. W. Sweldens, "The Lifting Scheme: A Custom-Design Construction of Biorthogonal Wavelets," Applied and Computational Harmonic Analysis, Vol. 3, No. 2, pp. 186-200, 1996

8. M. Hussain, I. Ullah, H. A. Aboalsamh, G. Muhammad, G. Bebis, and A. M. Mirza, "Gender Recognition from Face Images with Dyadic Wavelet Transform and Local Binary Pattern," International Journal on Artificial Intelligence Tools, Vol. 22, No. 6, pp. 1318-1336, 2014

9. G. J. Tu and H. Karstoft, "Logarithmic Dyadic Wavelet Transform with its Applications in Edge Detection and Reconstruction," Applied Soft Computing Journal, Vol. 26, No. 8, pp. 193-201, 2015

10. T. Abdukirim, S. Takano, and K. Niijima, "Construction of Spline Dyadic Wavelet Filters," Research Report on Information Science and Electrical Engineering of Kyushu University, Vol. 7, No. 1, pp. 1-6, 2002

11. K. Singh, D. K. Vishwakarma, G. S. Walia, and R. Kapoor, "Contrast Enhancement via Texture Region Based Histogram Equalization," Journal of Modern Optics, Vol. 63, No. 15, pp. 1-7, 2016

12. B. L. Sturm and S. Mallat, "A Wavelet Tour of Signal Processing," Computer Music Journal, Vol. 31, No. 3, pp. 83-85, 2014

13. D. D. Han, T. C. Zhang, and J. Zhang, "Research and Implementation of an Improved Canny Edge Detection Algorithm," Key Engineering Materials, Vol. 572, No. 1, pp. 566-569, 2014

14. S. Wang, X. Wu, and T. Liu, "Multiscale Ncut based on Dyadic Wavelet Transform," Computer Engineering and Applications, Vol. 51, No. 13, pp. 9-14, 2015

15. S. Mallat and S. Zhong, "Characterization of Signals from Multiscale Edges," IEEE Transactions on Pattern Analysis and Machine Intelligence, Vol. 14, No. 7, pp. 710-732, 1992 